\title{
Morphological and immunohistological characteristics of follicular-compact thyroid carcinoma in dog
}

\author{
Rafal Ciaputa ${ }^{1}$, Marcin Nowak ${ }^{1}$, Malgorzata Kandefer-Gola ${ }^{1}$, Katarzyna Dziedzic ${ }^{1}$, \\ Pawel Halupka $^{1}$, Bartosz Pula ${ }^{2}$, Maciej Kielbowicz ${ }^{3}$ \\ ${ }^{1}$ Department of Pathology, Faculty of Veterinary Medicine, University of Environmental and Life \\ Sciences, Wroclaw, Poland \\ ${ }^{2}$ Department of Histology and Embryology, Wroclaw Medical University, Wroclaw, Poland \\ ${ }^{3}$ Department of Surgery, Faculty of Veterinary Medicine, University of Environmental and Life \\ Sciences, Wroclaw, Poland
}

\begin{abstract}
The case of a 14-year-old mongrel dog with a thyroid tumor treated by thyreoidectomy is described. The resected tumor was subjected to a detailed morphological and immunohistochemical analysis utilizing antibodies directed against thyroglobulin, calcitonin, chromogranin A, cytokeratin 19, thyroid transcription factor-1, CD31, Ki-67 and minichromosome maintenance protein 3. Expression level of the abovementioned antigens allowed to characterize the resected tumor as thyroid follicular-compact carcinoma. Common application of immunohistochemistry may increase the diagnosis precision and efficacy of thyroid tumor treatment in dogs. (Folia Histochemica et Cytobiologica 2014, Vol. 52, No. 2, 157-161)
\end{abstract}

Key words: thyroid cancer; follicular-compact cancer; dog

\section{Introduction}

Canine thyroid tumors account from $1 \%$ to $3 \%$ of all neoplasias in the species and mostly involve a single lobe [1]. Morphological traits of the tumors allow to distinguish well-differentiated, poorly-differentiated and undifferentiated (seldom seen) thyroid carcinomas. The well-differentiated malignant tumors include follicular carcinomas with an evident follicular structure and more or less solid cellular structure (follicular-compact cellular carcinoma, compact cellular carcinoma), and medullary carcinomas developing from parafollicular $\mathrm{C}$ cells $[2,3]$. Precise diagnosis of a tumor in some circumstances requires ancillary methods to the routine hematoxylin and eosin $(\mathrm{H} \& \mathrm{E})$

\footnotetext{
Correspondence address: R. Ciaputa,

Department of Pathology, Faculty of Veterinary Medicine,

University of Environmental and Life Sciences,

Norwida Str. 31, 50-375 Wroclaw, Poland

tel.: +48 7132052 56; fax: +48 7132054 14;

e-mail: rafal.ciaputa@up.wroc.pl
}

staining. Therefore, immunohistochemical techniques must be often additionally implemented to make the final diagnosis. Immunohistochemistry (IHC) is not only useful for confirmation of the preliminary diagnosis of canine thyroid tumors, but it also allows to predict prognosis. Regrettably, respective scientific reports on the subject remain relatively scanty as in veterinarian practice IHC does not belong to the routinely performed techniques. In the current report the isolated tumor was subjected to an accurate morphological and IHC analysis. The obtained detailed characteristics enriched the currently restricted knowledge on the application of various markers in diagnosis of canine thyroid tumors.

\section{Clinical examination and handling}

The 14-year-old mongrel dog with transplacable tumor of the neck was referred to the Veterinarian Outpatient Clinic of the Department of Surgery. The trachea manifested tenderness upon physical examination. Ultrasound examination demonstrated a non-uniform, well separated and blood supplied 

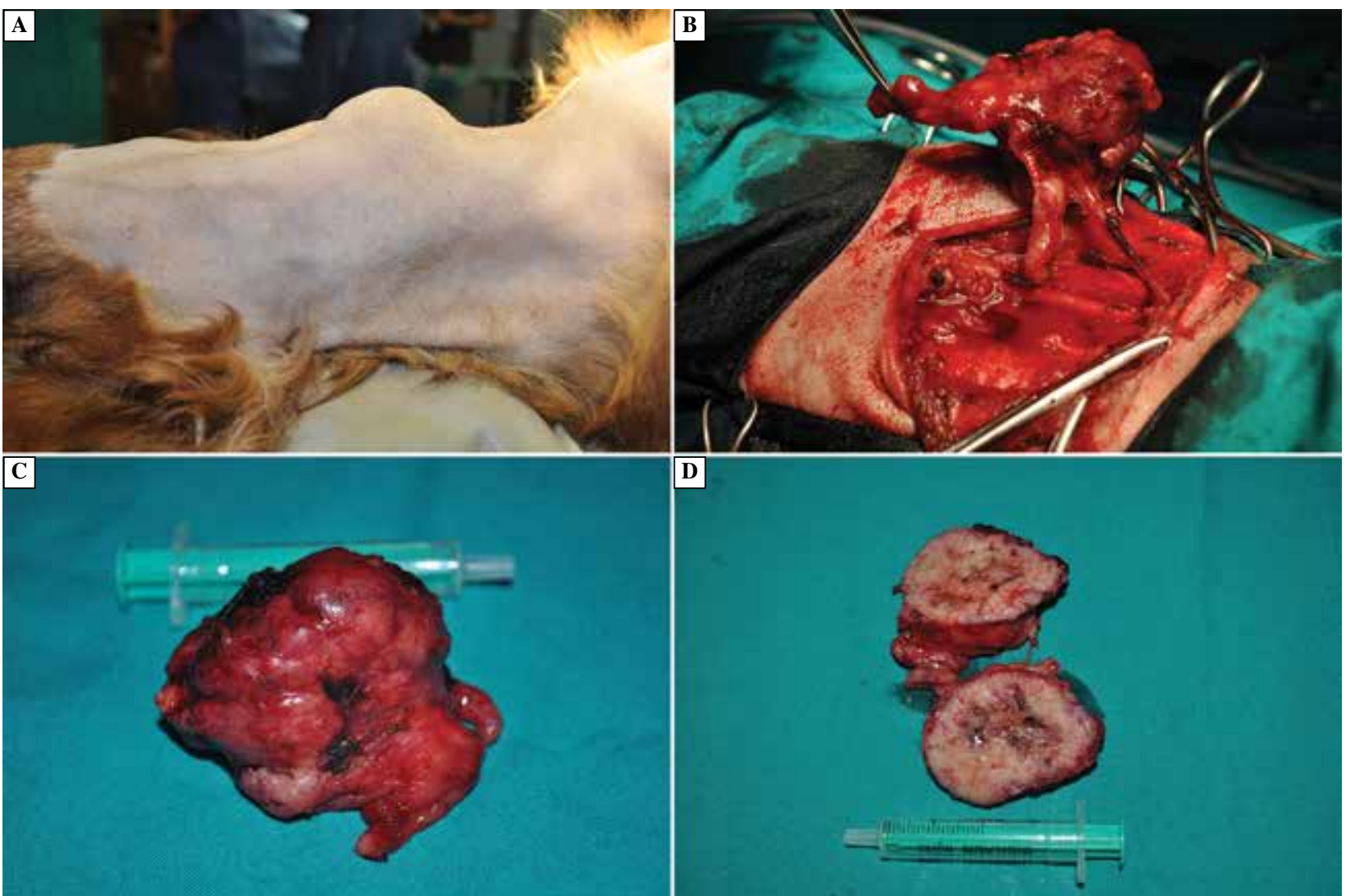

Figure 1. Mongrel dog with thyroid tumor. A. Transplacable tumor of the cervical regio. B. Intraoperative view. C. Macroscopic image of the excised tumor. D. Cross-section of the excised tumor with central necrotic region

tumor in projection of the left thyroid lobe, around $4.5 \times 3.5 \times 3.5 \mathrm{~cm}$ in size. No lymphadenopathy was observed in surrounding cervical tissues. In the chest $\mathrm{X}$-ray no pulmonary lesion suspicious of metastases were found.

Thyroidectomy confirmed the presence of an oval tumor, separated from vicinity by a thin capsule. The organ was removed in toto and transferred to the Department of Pathology (Figure 1A). Cross-section of the tumor disclosed centrally located focus of necrosis (Figure 1B). Due to the absence of pulmonary metastases or infiltration of the surrounding tissues the prognosis was defined as favourable.

\section{Diagnostic procedure}

The excised tumor was examined on H\&E- and IHC-stained paraffin sections. Immunohistochemistry was performed utilizing following murine anti-human monoclonal antibodies (at respective concentrations applied for $1 \mathrm{~h}$ at room temperature) directed against following antigens: cytokeratin 19 (CK-19; clone RCK108; 1:100), thyroid transcription factor (TTF-1; clone 8G7G3/1; 1:100), Ki-67 antigen (clone MIB-1;
1:100), minichromosome maintenance protein 3 (MCM3; clone 101; 1:50, Novocastra, Newcastle, UK), CD31 (clone JC70A; 1:100), thyroglobulin (Tg; clone DAK-Tg6; 1:100) and chromogranin A (clone DAK-A3; 1:100). For calcitonin detection polyclonal rabbit anti-human antibody (1:400) was used. The studied antigens were visualized using secondary biotinylated antibodies and streptavidin-biotinylated peroxidase complex (LSAB2, HRP) according to the manufacturer's instructions. The 3,3'-diaminobenzidine (DAB; for $7 \mathrm{~min}$, room temperature) was used as a substrate for the reaction. All the sections were counterstained with Meyer's hematoxylin. All the reagents and antibodies (except the MCM3 antibody) were obtained from DakoCytomation (Glostrup, Denmark).

\section{Diagnosis}

Histological evaluation of $\mathrm{H} \&$ E-stained sections demonstrated a fully obliterated organ structure with regions of neoplastic hyperplasia. The majority of the tumor was composed of solid bands of tumor cells with large irregular in shape nuclei, containing granular chromatin. The bands were separated from 


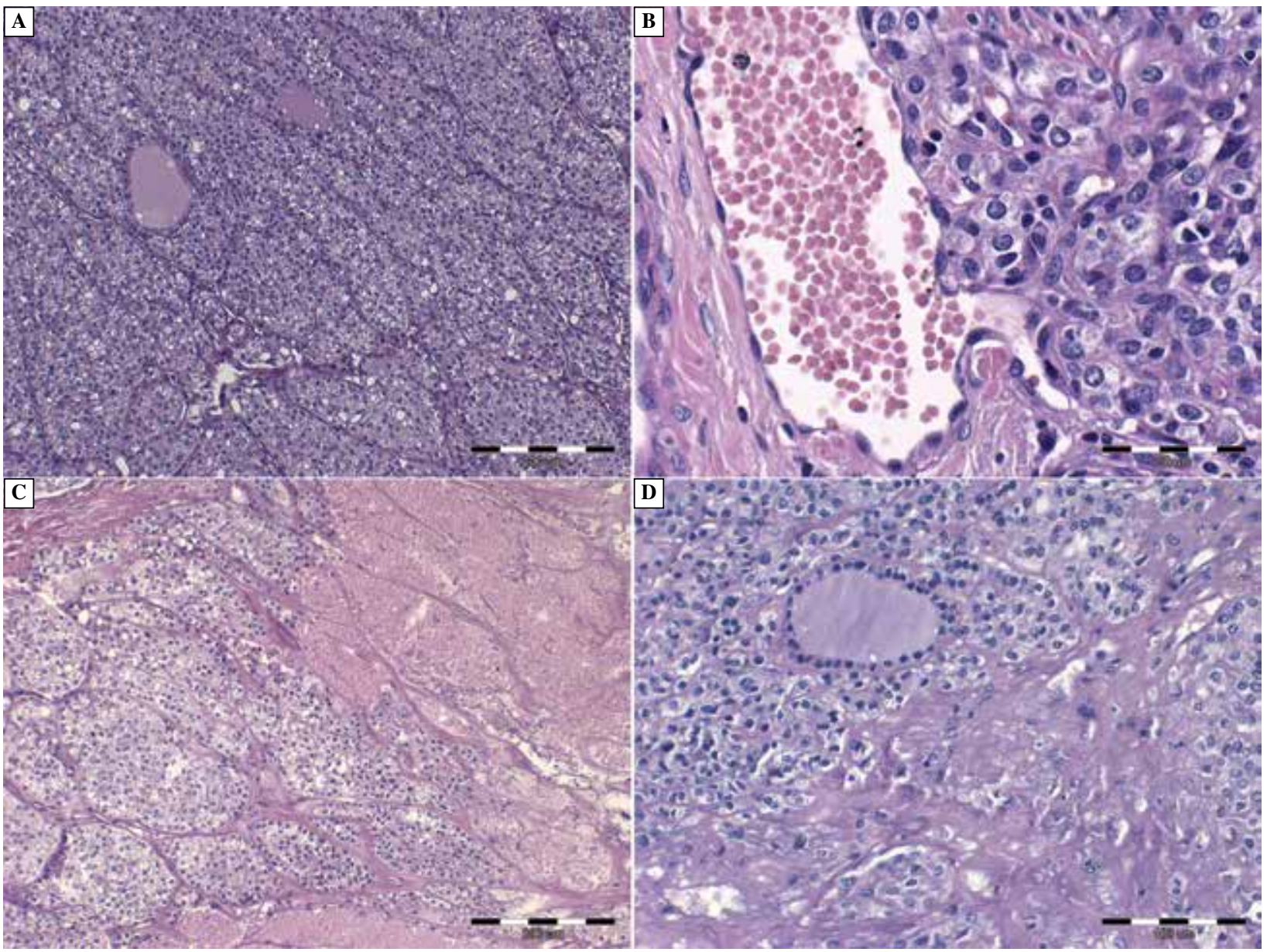

Figure 2. Dog, thyroid tumor, H\&E staining. A. Region with a compact structure of undifferentiated cells with preserved individual normal vesicles. B. Blood vessel infiltrated by neoplastic cells. C. Interruption of tumor capsule. D. Dissolving necrotic masses of neoplastic thyroid cells

each other by fibrous septa of a variable thickness, containing thin-wall blood vessels. Occasionally, individual, small colloid-filled vesicles were encountered (Figure 2A). The neoplastic cells were found to infiltrate and damage the walls of blood vessels (Figure 2B) as well as interruption of tumor capsule was observed (Figure 2C). In several parts of the tumor haemorrhagic foci and necrotic regions of various size were observed (Figure 2D).

The IHC examination disclosed variable levels of expression of examined markers. A definitely highest expression level was noted in cases of thyroglobulin, cytokeratin-19 (both cytoplasmic localization), while a lower nuclear TTF-1, Ki-67 (32\% of positive cells) and MCM3 (35\% of positive cells) expression level was demonstrated (Figure 3). No expression of calcitonin or chromogranin A was noted in the tumor cells. Using the anti-CD31 antibody, infiltration of adjacent vessels by tumor cells observed in H\&E-slides was confirmed (Figure 3).
The histopathological and IHC patterns allowed to diagnose a thyroid carcinoma of a vesicular-compact type. Adenoma, medullary carcinoma, mixed vesiculomedullary thyroid carcinoma, carotid body tumor, soft-tissue sarcoma and haemangiopericytoma were taken into consideration in the differential diagnosis of the above described tumor.

\section{Discussion}

Thyroid tumors in dogs account for around 10-15\% of all neoplasias within the head and neck region, and $90 \%$ of them are diagnosed as carcinomas. Already at the moment of diagnosis $16 \%$ to $38 \%$ patients carry visible metastases. Although no sex prevalence was detected, age-related (10 to 15 years of age) and breed-linked (Beagles, Golden retriever, Syberian husky) predispositions were documented [4, 5]. In dogs an extensive practical significance is linked to clinical evaluation of a tumor according to guidelines 


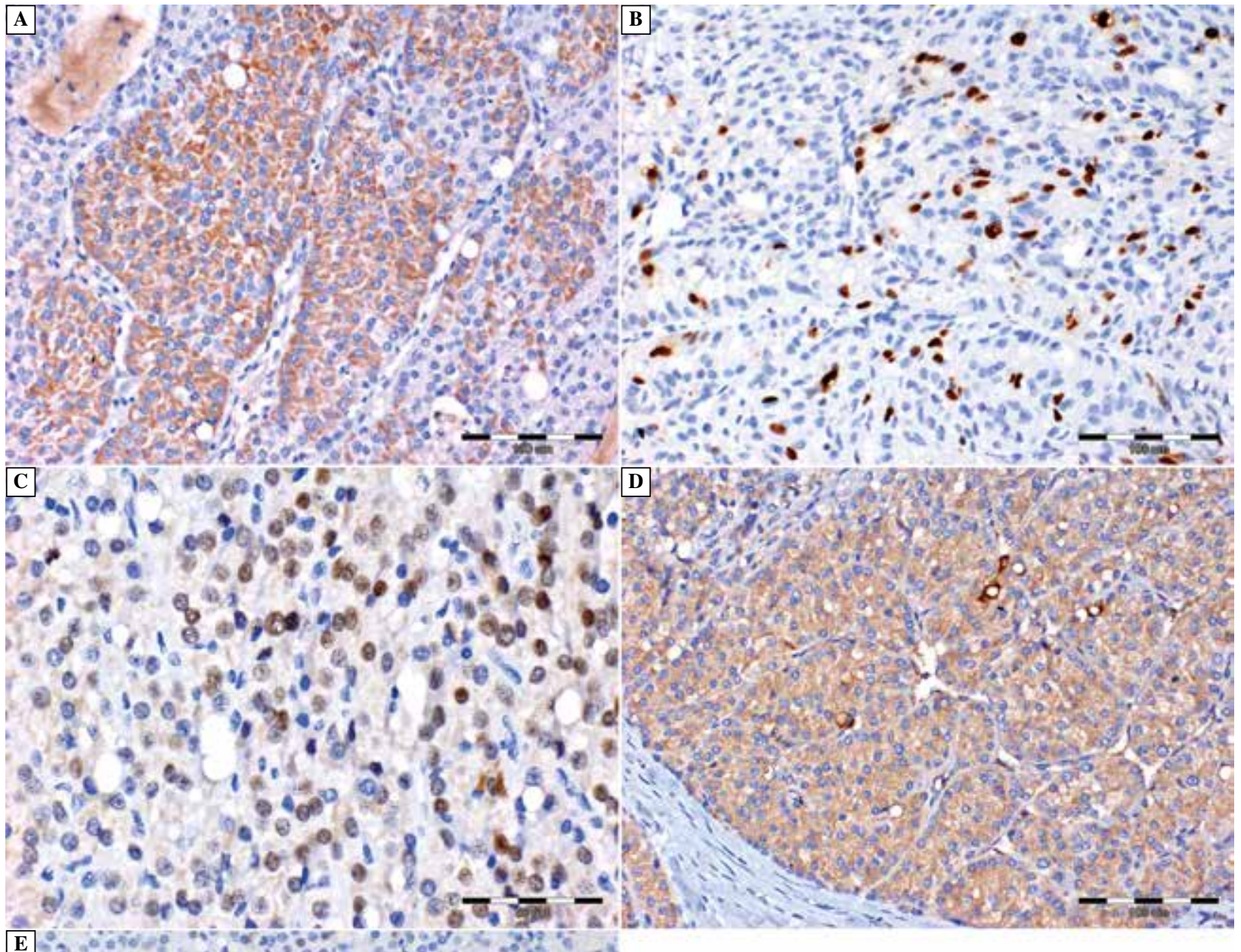

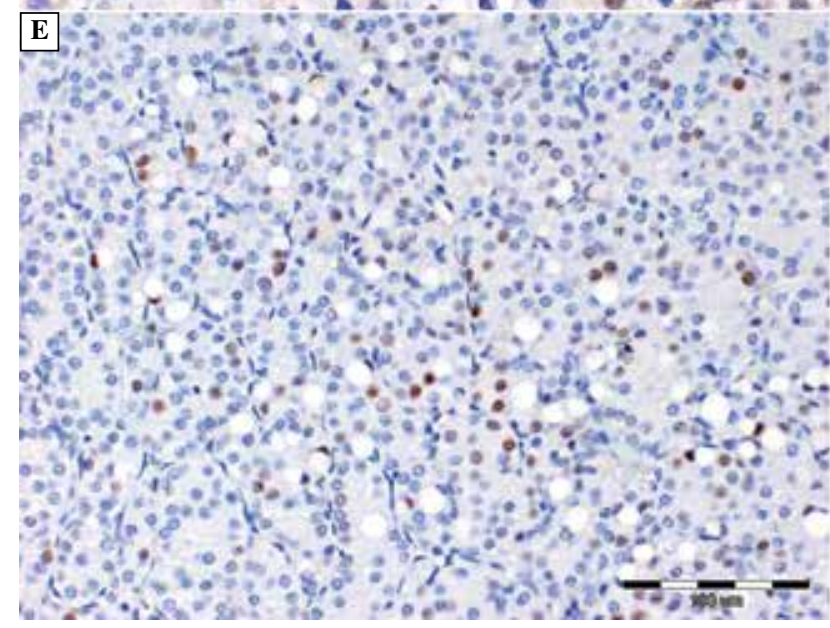

of World Health Organization (WHO) (involving i.a., tumor size, transplacebility) what provides basis for selection of the most accurate therapeutic management [1]. In cases of skin-transplacable cancers thyroidectomy represents the approach of choice. However, upon detection of extensive tumors which infiltrate surrounding tissues external beam therapy or radioactive iodine-131 $\left({ }^{131} \mathrm{I}\right)$ therapy are recommended [4]. Histological evaluation of canine tumor types seems
Figure 3. Expression of selected markers in canine thyroid neoplastic cells. High expression of cytokeratin-19 (A), Ki-67 (B), TTF-1 (C), thyroglobulin (D), MCM-3 (E) in the cells of thyroid carcinoma to be easier than in humans due to lower variability of forms demonstrated by the lesions [2]. Nevertheless, IHC evaluation is indispensable for establishment of a proper diagnosis of the tumor subtype. Such tests are crucial in confirmation of thyroid origin of carcinomas developing in the head and neck region in cats or dogs [6-8]. It is worth to mention that introduction of IHC techniques into diagnosis of canine thyroid tumors resulted in an increasing frequency of medullary 
carcinoma diagnoses, earlier thought to represent a very rare neoplasia [8]. Lines of evidence suggest that application of calcitonin, thyroglobulin, TTF-1 and chromogranin A as IHC markers is useful in differential diagnosis of canine thyroid lesions [8-10]. In this report, the set of markers has been supplemented with CK-19, $\mathrm{Ki}-67$ antigen and MCM3 protein. CK-19, in line with galectin-3, Ret oncoprotein, HBME-1 may be useful in distinguishing malignant from benign lesions since in human carcinomas the reaction product is pronounced and localized in a diffuse manner. First of all, CK-19 is a useful marker in confirmation of epithelial origin of the tumor [11]. The extent of immunostaining is higher in papillary carcinoma, however CK-19 is present in all cases of follicular carcinoma at least focally [12]. The high mitotic potential of the analyzed tumor was confirmed by the high expression of Ki-67 antigen in cancer cells. Moreover, a pronounced expression of MCM3 in tumor cells (comparable to that of the Ki-67 antigen) was demonstrated. In some studies MCM3 was shown, to be an useful marker for determination of cell proliferative potential in canine tumors of various histological types [13]. The CD31 staining confirmed the observed infitration of tumor capsule by cancer cells, as the latter were also observed in lumen of the CD31-positive vessels. The studied canine thyroid carcinomas has manifested a selective reactivity with the applied antibodies. The selectivity has corresponded to cellular structure of the tumor, typical for thyroid carcinomas of a vesicular origin. The negative results of IHC directed towards detection of chromogranin $A$ and calcitonin have been expected since the compounds represent markers of medullary carcinomas (originating from C cells) $[8,9,14]$. The accurate diagnosis of carcinoma type in dogs may be of prognostic significance since medullary carcinomas in these animals used to be well encapsulated, less invasive and manifest lower tendency to develop metastases as compared to vesicular carcinomas [8]. Apart from assistance in stratifying the prognosis, an accurate diagnosis of canine thyroid carcinoma type might prove usefulness in therapy, similarly to the situation seen in humans. In studies of Liptak et al. therapy with radioactive iodine yielded encouraging results in dogs subjected earlier to thyroidectomy (mean survival 30-34 months) [1]. However, the authors did not mention whether the removed carcinomas were subjected to an accurate classification of types. It might be expected that tumor positively reacting to such a treatment represented follicular carcinomas since only follicular cells are capable of iodine absorption [14]. In cases of medullary carcinomas, originating from iodine not absorbing parafollicular cells, such a treatment would be pointless.

\section{Conclusion}

The presented case report aimed at presenting the potential utility of immunohistochemistry in the diagnosis of a thyroid tumor in dog. The selected panel of antibodies allowed for a precise diagnosis of a follicular-compact carcinoma. Precise identification of the type of cancer can increase the effectiveness of the treatment and yield prognostic significance.

\section{References}

1. Liptak JM. Canine thyroid carcinoma. Clin Tech Small Anim Pract. 2007;22:75-81.

2. Capen C.C. Thyroid gland. In: Jubb, Kennedy and Palmer's Pathology of Domestic Animal. Vol 3, 5th Ed. Saunders Ltd., Philadelphia; 2007:396-402.

3. Kiupel M, Capen C, Miller M, Smedley R. Histological Classification of Tumors of the Endocrine System of Domestic Animals. Second Series Vol XII. World Health Organization, International Histological Classification of Tumors of Domestic Animals. Armed Forces Institute of Pathology, Washington, D.C.; 2008:25-39.

4. Campos M, Peremans K, Duchateau L et al. Effect of recombinant human TSH on the uptake of radioactive iodine (123I) by the thyroid gland in healthy beagles. Domest Anim Endocrinol. 2010;39:215-221.

5. Wucherer KL, Wilke V. Thyroid cancer in dogs: an update based on 638 cases (1995-2005). J Am Anim Hosp Assoc. 2010;46:249-254.

6. Min-Hee K, Dae-Young K, Hee-Myung P. Ectopic thyroid carcinoma infiltrating the right atrium of the heart in a dog. Can Vet J. 2012;53:177-181.

7. Knowles S, Uhl EW, Blas-Machado U, Butler MA. Intrapericardial ectopic thyroid carcinoma in a cat. J Vet Diagn Invest. 2010;22:1010-1013.

8. Allison RW, Maddux JM. Subcutaneous Glandular Tissue: Mammary, Salivary, Thyroid and Parathyroid. In: Cowell RL, Tyler RD, Meinkoth JH, DeNicola DB, Diagnostic Cytology and Hematology of the Dog and Cat. 3rd Ed, Mosby Elsevier, St. Louis; 2008:121-125.

9. Patnaik AK, Lieberman PH. Gross, Histologic, Cytochemical, and Immunocytochemical Study of Medullary Thyroid Carcinoma in Sixteen Dogs. Vet Pathol. 1991;28:223-233.

10. Ramos-Vara JA, Miller MA, Johnson GC, Pace LW. Immunohistochemical Detection of Thyroid Transcription Factor-1, Thyroglobulin, and Calcitonin in Canine Normal, Hyperplastic, and Neoplastic Thyroid Gland. Vet Pathol. 2002;39:480-487.

11. Saleh HA, Jin B, Barnwell J, Alzohaili O. Utility of immunohistochemical markers in differentiating benign from malignant follicular-derived thyroid nodules. Diagnostic Pathology. 2010;5:10.

12. Dabbs DJ. Diagnostic Immunohistochemistry. 2nd Ed. Churchill Livingstone Elsevier, Philadelphia, 2006;267-276.

13. Nowak M., Madej JA, Dziegiel P. Correlation Between MCM3 Protein Expression and Grade of Malignancy in Mammary Adenocarcinomas and Soft Tissue Fibrosarcomas in Dogs. In vivo. 2009;23:49-54.

14. Chan JKC. Tumors of the thyroid and parathyroid glands. In: Fletcher CDM. Diagnostic histopathology of tumors. 2nd ed. Churchill Livingstone Elsevier, Philadelphia; 2007:997-1081.

Submitted: 25 June, 2013

Accepted after reviews: 15 May, 2014 